\title{
AN EXPERIMENTAL METHOD FOR THE PRODUCTION OF VIBRATIONS.
}

\author{
By C. V. Raman.
}

SyNOPSIS.

\begin{abstract}
Forced Vibration of strings; the Melde experiment. Improvements of Fleming's modification are described which enable the amplitude of the forced vibration to be varied, which minimize lateral vibration, and which make it possible to obtain linear, circular or elliptical vibration as desired. This improved apparatus is useful for studying or demonstrating the various types of motion possible for a stretched string. Five photographs illustrate the results which may be obtained.

Forced Vibration of strings. The phenomenon observed by Jones and Phelps, that when two or more different types of vibration are simultaneously obtained, the ratio of the number of ventrals egments may differ from the ratio of the frequencies of maintenance, is briefly discussed.
\end{abstract}

PROF. J. A. FLEMING ${ }^{1}$ has recently devised and described a very simple but ingenious and compact piece of apparatus to illustrate the close analogy existing between the transmission of mechanical vibrations along a loaded string and the propagation of alternating electric currents along a telephone cable having inductance coils inserted in it at equidistant intervals. For this purpose, the well-known method of producing forced vibrations on strings due to F. Melde is not suitable; for, when a long heavily loaded string is used, very large forks would be required, and the reaction of the string when under tension would generally result in stopping the fork even if the latter be electrically driven. Further, it is also desirable to have a means of altering the frequency of vibration which is not possible with Melde's method except by changing the fork. Prof. Fleming therefore replaced the fork in the Melde experiment by a small continuous-current electric motor, on the shaft of which a disk carrying an excentrically placed pin is fixed. The pin actuates a light crank shaft, which is connected at the other end to a rocking lever. The string is maintained in vibration by having its extremity attached to a small hook fixed to the crank-shaft. With an arrangement of this kind, it is possible to impose either a linear or circular vibratory movement on the extremity of the string as desired.

In experimenting with an apparatus of the type described above, the present author found that its usefulness is considerably enhanced by

${ }^{1}$ Physical Society, London, Proc., December, r913, p. 6r. 
certain improvements which he has made in it. In Fleming's apparatus, as illustrated in his paper, the excentric pin is permanently fixed to the disk carried on the shaft of the motor. The amplitude of the obligatory circular or linear vibration imposed on the extremity of the string is consequently invariable. This is a disadvantage, as in the practical use of the apparatus, it is often useful to be able to alter the amplitude of the obligatory motion quickly to any value, either large or small, as may be desired. Further, the author has found that with an apparatus of the Fleming type, when the shaft of the motor is in rapid rotation, the rocking lever and crank-shaft tend to develop vibrations in a direction transverse to their plane, and this introduces undesirable complications in the forced vibration of the extremity of the string. To remedy these defects and make the apparatus a real precision instrument for the study of vibrations, the design of the working parts has been modified as shown in Fig. I. The disk fixed to the shaft of the motor has a slotted plate screwed to it a little above the general surface, and the crank-pin is carried by a sliding piece which can be put at any desired point and fixed by a single half-turn of the screw-head shown in the figure. The rocking lever moves between well-oiled guides which prevent any lateral vibration, the whole being firmly bolted to the body of the motor as shown in the photograph of the apparatus (Fig. 2). Linear movements may be obtained by attaching the string to one or other of a number of holes in the rocking-lever. A slid-

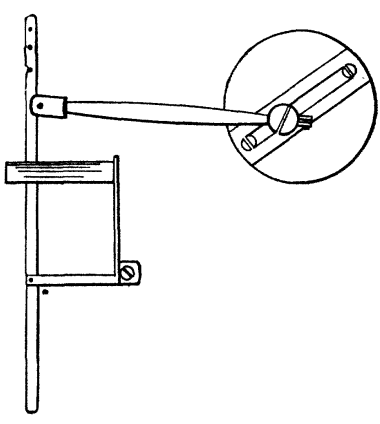

Fig. 1. ing piece on the crank-shaft (not shown in the figure) which carries a hook and can be fixed at any point enables either circular or elliptical vibrations to be imposed on the extremity of the string if desired.

The arrangement (modified as described above) has proved to be a most convenient and satisfactory piece of apparatus. It is especially useful in the experimental study or demonstration of the interesting types of vibration maintained by a variable tension, described by the author in previous communications in this Review. ${ }^{1}$ These types are obtained when the string is attached to the rocking-lever in such a manner that its oscillation is longitudinal to the string. Under these conditions, as has been shown in the papers quoted, the string may be set in vibration when its frequency in any of its natural modes is sufficiently nearly equal to any multiple of half the frequency of the variation of tension.

${ }^{1}$ Phys. Rev., December, 1912, and July, r9r4. 
Each of the possible modes has its own characteristic features, and as moreover, the frequency of vibration of the string depends on the number of ventral segments into which it divides up and may thus have simultaneously more than one value, a very large variety of different modes of vibration may be obtained at will by adjusting the tension or length of the string, or by altering the position of the crank-pin of the motor so as to increase or decrease the magnitude of the imposed variation of tension.

The motion of individual points on the string may be very conveniently studied by using a black cord with white dots woven in it as suggested by $A$. T. Jones and M. E. Phelps in a recent paper in this REview. ${ }^{1}$ The form of the figures thus observed reveals at a glance not only the relation of frequency between the longitudinal and transverse movements of any point on the string, but also their phase-relation which appreciably varies with the experimental conditions. Much longer and heavier strings can be used with this apparatus than with an electricallymaintained fork, and by lighting up with the beam from an electric arc, very effective demonstrations are possible. To illustrate the character of the phenomena observed, photographs of the motion of individual points on the string in the first five types of vibration have been secured and are reproduced in Figs. 3 to I2. Figs. 3 and 4 show the first type of vibration in which the ratio of frequencies is $\mathrm{I}: 2$, the phase-relation being slightly different in the two cases. In Figs. 5 and 6 the frequency relation is $2: 2$, the curve in Fig. 6 being a deformed ellipse. In Figs. 7,8 and 9 we have the third type in which the frequency ratio is $3: 2$, the difference in phase being specially marked in Fig. 9. The fourth type in which the frequency relation is $4: 2$ is shown in Figs. Io and II. The fifth type is shown in Fig. 12 , and exhibits the frequency relation $5: 2$.

It may be useful here to consider the explanation of the interesting phenomenon noticed by Jones and Phelps in their paper, ${ }^{2}$ that when two or more of the different types are simultaneously maintained, the ratio of the number of ventral segments into which the string respectively divides up often differs from the ratio of the frequencies of maintenance. For instance, it may be found in one case that the string divides up simultaneously into two and five segments either in the same plane or in perpendicular planes, the frequencies being respectively one half and three halves of the frequency of variation of tension. Apparent anomalies of this kind are due to the fact that we are here dealing with forced oscillations, and though the string practically vibrates in its normal

1 Phys. Rev., November, 1917.

2 Loc. cit. 
Physical Review, Vol. XIV., Second Series. November, I9I9.

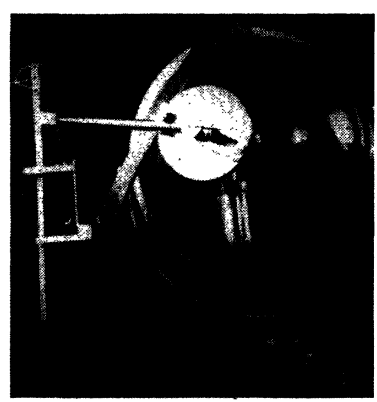

Fig. 2.

3
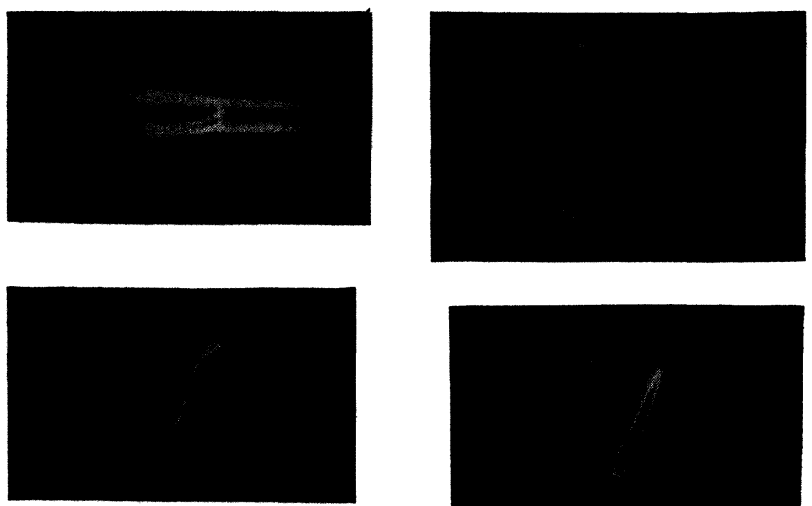

5
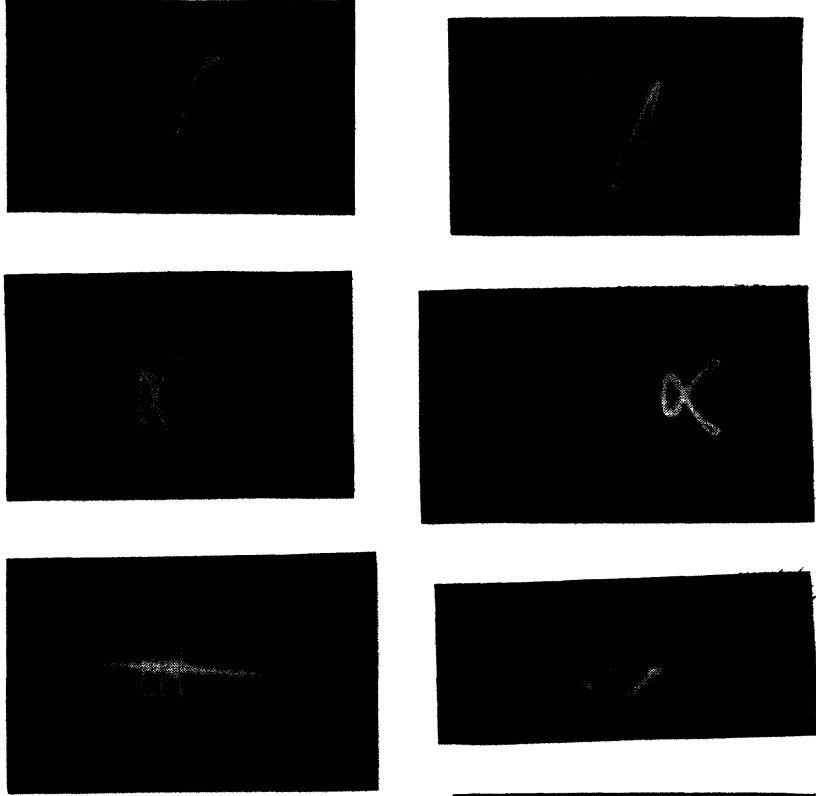

6

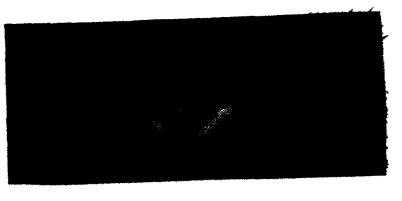

Io

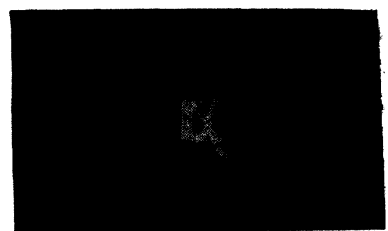

I 2

Figs. 3-12.

C. V. RAMAN. 
modes, the frequencies of vibration stand in fixed relations to the impressed forces and may, especially in the case of the first two or three types, differ very appreciably from the natural frequencies of oscillation. Maintenance is, in fact possible when the natural frequency lies anywhere within a certain range which includes the frequency of maintefiance, this range being considerable for the first type, and becoming smaller and smaller as we proceed up the series of types with higher frequencies of maintenance. The magnitudes of the ranges depend on the magnitude of the imposed variations of tension, as may be readily shown with the apparatus described in the present paper, by adjusting the position of the crank-pin, and thus increasing or decreasing the longitudinal motion of the end of the string. With very small variations of tension, the modes with the higher frequencies of maintenance are hardly obtainable, the resonances are much sharper, and the ranges even of the first few types become greatly restricted. With larger variations of tension, the ranges increase, and for the first few types may become very considerable. It will be found that large amplitudes may be obtained even without any exact adjustment for resonance.

The photographs reproduced with the paper were secured for the author by Mr. Rajendra Nath Ghosh, for whose assistance his thanks are due. Mr. Ghosh is now engaged in developing a detailed theory showing the manner in which the amplitudes and phases of the various types of vibration depend on the experimental conditions. The results will, it is hoped, be published in due course.

2I0, Bowbazar STreet,

Calcutta, India,

May 20, I9I9. 


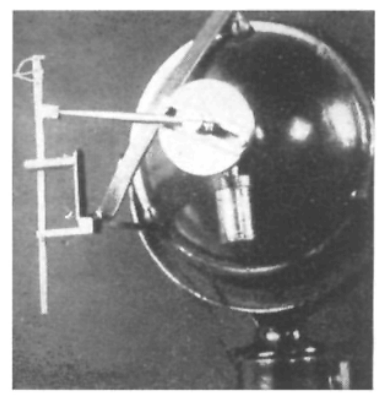

Fig. 2. 

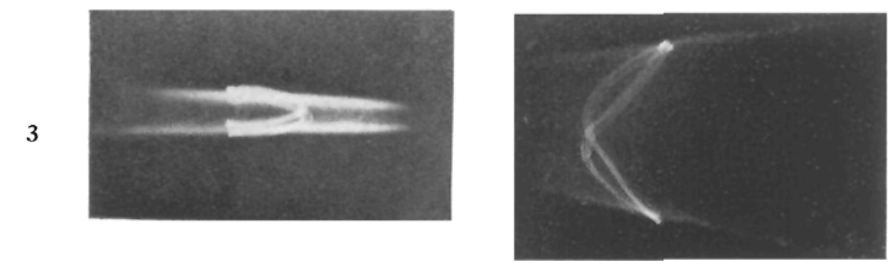

4
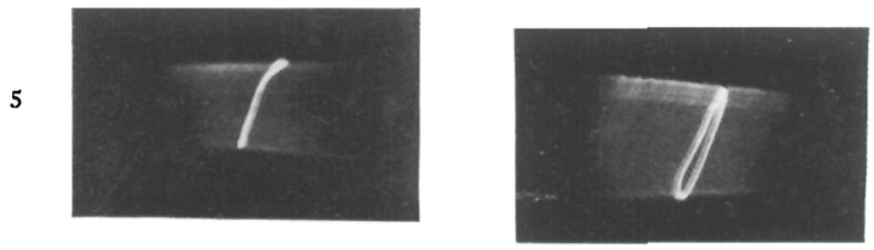

6
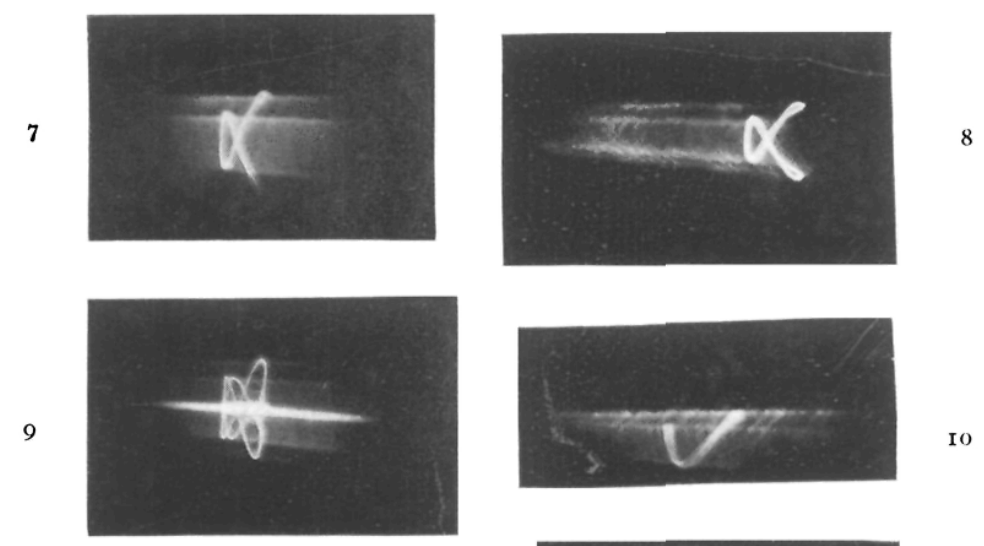

Io

II
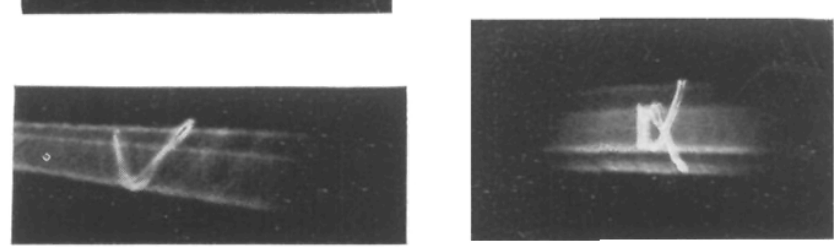

I 2

Figs. 3-12. 